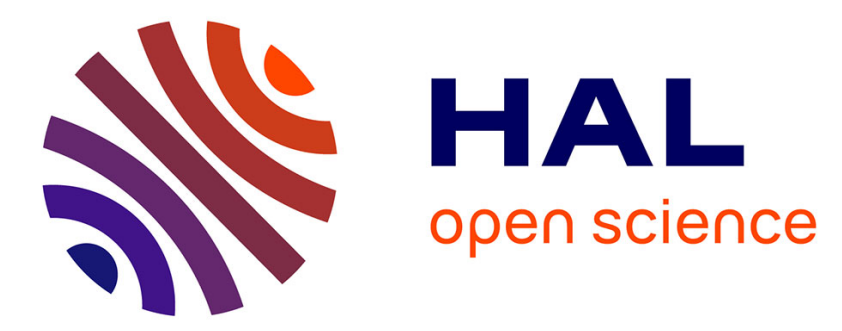

\title{
Acousto-optic imaging in liquids: a step towards in-vivo measurements
}

\author{
Pedro Santos, Michael Atlan, Benoit C. Forget, Emmanuel Bossy, Claude \\ Boccara, Michel Gross
}

\section{- To cite this version:}

Pedro Santos, Michael Atlan, Benoit C. Forget, Emmanuel Bossy, Claude Boccara, et al.. Acoustooptic imaging in liquids: a step towards in-vivo measurements. The Seventh Conference on Biomedical Thermoacoustics, Optoacoustics, and Acousto-optics, Mar 2006, United States. pp.608613, 10.1117/12.641676 . hal-00261656

\section{HAL Id: hal-00261656 \\ https://hal.science/hal-00261656}

Submitted on 14 Mar 2008

HAL is a multi-disciplinary open access archive for the deposit and dissemination of scientific research documents, whether they are published or not. The documents may come from teaching and research institutions in France or abroad, or from public or private research centers.
L'archive ouverte pluridisciplinaire HAL, est destinée au dépôt et à la diffusion de documents scientifiques de niveau recherche, publiés ou non, émanant des établissements d'enseignement et de recherche français ou étrangers, des laboratoires publics ou privés. 


\title{
Acousto-optic imaging in liquids : a step towards in-vivo measurements
}

\author{
Pedro Santos ${ }^{a}$, Michael Atlan ${ }^{a}$, Benoît C. Forget ${ }^{a}$, Emmanuel Bossy $^{a}$, A. Claude Boccara ${ }^{a}$ and \\ Michel Gross ${ }^{b}$ \\ ${ }^{a}$ Laboratoire d'Optique, ESPCI, CNRS UPRA0005, \\ Université Pierre et Marie Curie, 10 rue Vauquelin 75231 Paris cedex 05. France \\ ${ }^{b}$ Laboratoire Kastler-Brossel, Département de Physique de l'Ecole Normale Supérieure, \\ UMR 8552 (ENS, CNRS, UMPC), 10 rue Lhomond 75231 Paris cedex 05. France
}

\begin{abstract}
The aim of this paper is to show that we can perform acousto-optical signal acquisition of one datapoint (or voxel of a 3D image) in a very short time $(2-4 \mathrm{~ms})$, in order to overcome the speckle decorrelation effect. To demonstrate this, we have performed experiments in in dynamic scattering media such as liquids. We will show that we can work with pulsed wave ultrasound, to reduce the sound irradiation duration in order to be compatible with safety limits. These are significant steps towards in-vivo experiments.
\end{abstract}

Keywords: Medical and biological imaging, Speckle interferometry, Turbid media, Ultrasound, Holographic interferometry

\section{INTRODUCTION}

Acousto-optic imaging ${ }^{1-4}$ aims at obtaining images of optical contrast in centimeter thick biological tissues with the millimeter spatial resolution of ultrasound imaging. It is based on the modulation of the photon travel paths within the area of interaction with the focused ultrasound beam. This process is often referred to as "photon tagging". The detection of tagged photons is difficult : the signal is weak, spatially incoherent and since the detection schemes are usually based on a modulation of the speckle intensity they require that this pattern remain correlated throughout the whole measurement time. In the case of in-vivo measurements, blood flow limits this correlation time to roughly $1 \mathrm{~ms}^{5,6}$ Last but not least, the level of applied laser light and ultrasound must comply with safety regulations.

The heterodyne parallel speckle detection scheme we have developed allows to detect tagged photons with optimal (shot noise limited) sensitivity. ${ }^{7,8}$ Using either a pulse or a pseudo random phase modulation of the ultrasound and the reference beam it is possible to obtain acousto-optic images in dynamic scattering media at speeds (a few ms per voxel) compatible with future application to in-vivo imaging.

\section{HETERODYNE PARALLEL SPECKLE DETECTION}

The experimental setup is represented in figure 1. The setup itself as well as the experimental methodology of digital holography and heterodyne detection applied to AO imaging have been described previously ${ }^{7,9}$ and we will only briefly recall them here. As seen in figure 1, the setup is basically a Mach-Zender interferometer in which the the reference beam is frequency shifted by acousto-optic shifters (modulators). This is done in order to ensure that the static interference pattern recorded by the CCD or CMOS camera results from the interaction of the reference field and the so-called "tagged-photons" ${ }^{2}$ coming from the diffusing sample which have been

\footnotetext{
Further author information: (Send correspondence to Benoît C. Forget)

Benoît C. Forget : E-mail: forget@optique.espci.fr, Telephone: +33 (0)1 40794590

Michael Atlan : E-mail: atlan@optique.espci.fr

François Ramaz : E-mail: ramaz@optique.espci.fr

A. Claude Boccara : E-mail: boccara@optique.espci.fr

Michel Gross : E-mail: gross@lkb.ens.fr
} 
frequency shifted by the same amount amount through the acousto-optic effect. To achieve better detection the reference is frequency shifted by an extra amount in order to perform parallel lock-in detection ${ }^{10}$ with the CCD or CMOS camera. To further improve the signal to noise, a spatial filter (slit) is introduced at the output side of the diffusing sample and the reference beam is shifted to perform off-axis holography. ${ }^{11}$

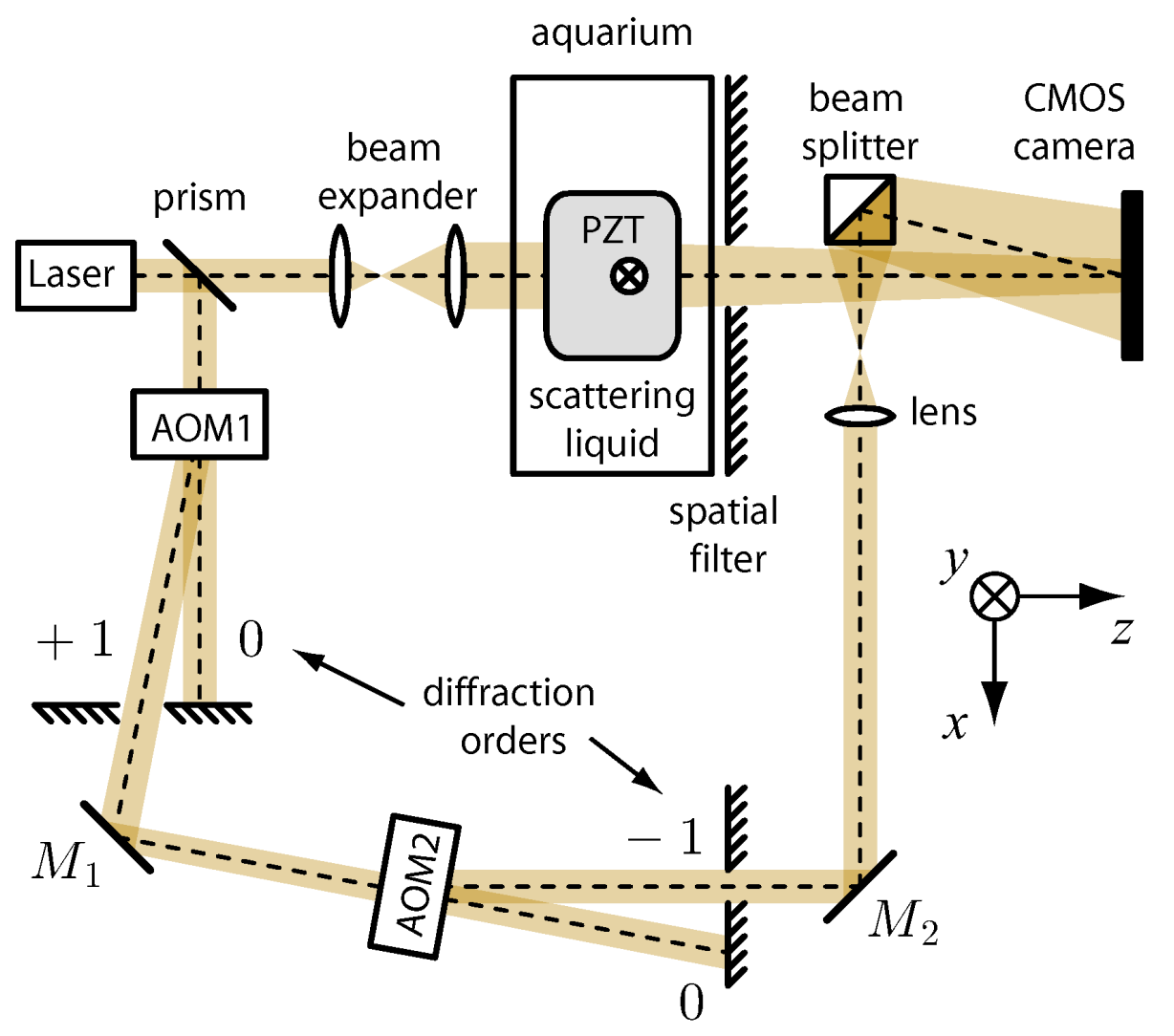

Figure 1. Setup. Near-infrared light (wavelength $\lambda_{L}=780 \mathrm{~nm}$, optical frequency $f_{L}$ ) is provided by a CW, single axial mode $400 \mathrm{~mW}$ output power titanium:sapphire laser (Coherent, MBR 110). The light path is split into a reference (local oscillator, LO) and an object arm, by a beam splitter. In the object arm, a set of lenses (not shown) expands the beam illuminating the sample. The sample is immersed in a transparent water tank, set on a 1D displacement stage. Transmitted light passes through a spatial filter (SF) made of a $3 \times 100 \mathrm{~mm}$ rectangular diaphragm. Two acousto-optic modulators (AOM1,2: Crystal Technologies, diffraction efficiency: 50\%) are placed in the reference arm, to shift the LO optical frequency $f_{L O}=f_{L}+f_{\mathrm{AOM} 1}-f_{\mathrm{AOM} 2}$, where $f_{\mathrm{AOM} 1,2}$ are the frequencies of signals sent to AOM1,2 . A $10 \mathrm{~mm}$ focal length lens is placed in the reference arm in order to create a slightly off-axis $\left(\theta \approx 1^{\circ}\right.$ tilt angle) virtual source point in the SF plane. A 10 bit, 1 Megapixel (square pixels, pixel size: $d=17.5 \mu \mathrm{m}$ ) CMOS camera (HSS4, LaVision) is set at a distance $L=40 \mathrm{~cm}$ from SF, facing the aperture, recording the interference pattern of light from both arms, at framerate $f_{C}$. An acoustic transducer (Panametrics Accuscan A395S-SU, focal length: $68 \mathrm{~mm}$ ) provides the ultrasonic pressure wave at the frequency $f_{A}=2.25 \mathrm{MHz}, 0.25 \mathrm{MPa}$ at focal point.

\section{PULSED AND PSEUDO RANDOM MODULATION TECHNIQUES}

To address the problem of spatial resolution along the ultrasonic axis $z$ (axial resolution), Wang et al. developed frequency-swept $\mathrm{AO}$ imaging with a monodetector. ${ }^{12,13}$ Forget et al. ${ }^{14}$ have extended this chirp technique to PSD by recording a sequence of CCD images while sweeping the frequency of the US. These chirp techniques require that the speckle remains correlated throughout the duration of the frequency chirp (several seconds), and are thus incompatible with in vivo tomography, because the speckle looses its memory in a time ( $1 \mathrm{~ms})$ much shorter that the chirp duration $(\sim 10 s)$. 


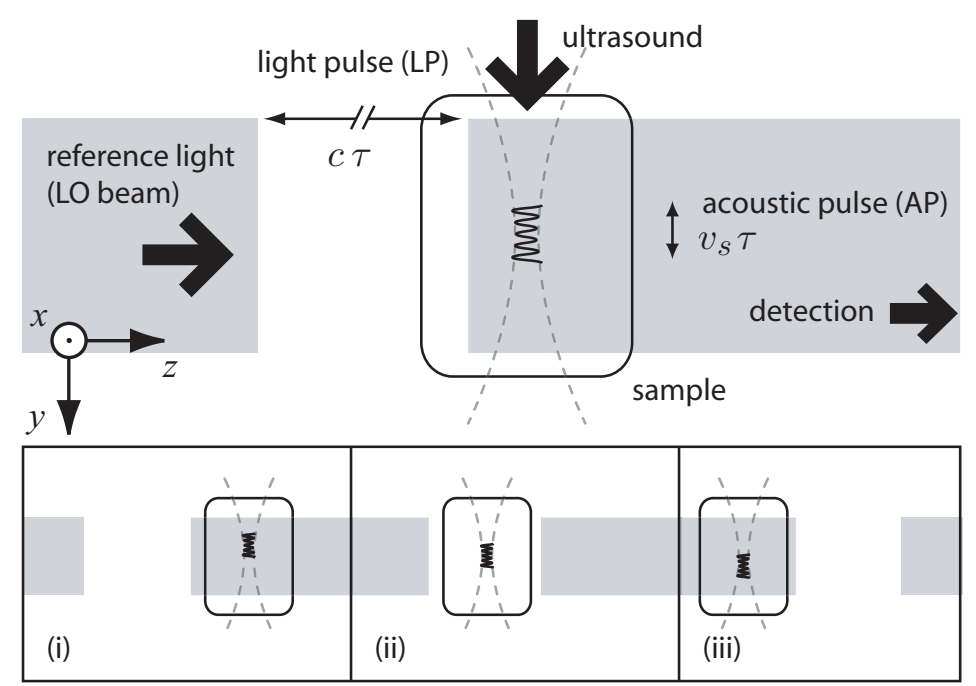

Figure 2. Stroboscopic detection of the acoustic pulse AP (pulse length $v_{s} \tau$ where $v_{s} \simeq 1500 \mathrm{~m} / \mathrm{s}$ is sound velocity) by the LO beam light pulse LP (pulse length $c \tau$ where $c$ is light velocity). (i) the AP propagates in the sample while the LO beam is turned off. (ii) AP and LP overlap within the AO volume of interest. Heterodyne amplification and detection of ultrasound-tagged photons occurs. (iii) the LO beam is turned off until the next AP reaches the AO volume.

To get high axial resolution in vivo, Lev and $\mathrm{Sfez}^{5,15}$ use continuous-wave (CW) illumination and pulsed-wave (PW) ultrasound. However, the SNR is intrinsically low because they work with a single detector of a low optical throughput. We have adapted our parallel detection technique to accommodate pulsed-wave ultrasound. ${ }^{9}$ The principle is described in figure 2 .

The idea is to gate one of the optical beam in order to flash the propagating acoustic pulse at a specific location along axis $y$ (defined in figure 2). To better illustrate the technique, in figure 2 the object beam is gated, but for experiments it is more practical to gate the reference beam. The interference pattern is present (and recorded by the CCD or CMOS camera) only for a short time corresponding to the position of the acoustic pulse at this time in the media. By introducing a controllable delay between the launch of the acoustic and the reference light beam pulses it is possible to scan the positions along the propagation axis.

The axial resolution is clearly determined by the convolution product of the two time gates (acoustical and optical). As in can be seen in ref., ${ }^{9}$ increasing the pulse duration degrades the spatial resolution resolution (as well as the contrast which is intrinsically linked to the resolution). This has led us to propose a new scheme to obtain axial resolution based on pseudo random phase modulation. ${ }^{16}$

Consider again the interference pattern recorded by the camera, expressed as the product of the object field and the conjugate reference field, but this time we replace the gated sine phase modulation induced by the a pseudo random phase modulation $f_{r n d}(t)$ :

$$
I=O \times R^{*}=I_{0} e^{j f_{r n d}(t-z / v)} e^{-j f_{r n d}(t-\tau)}
$$

We must now take into account that the camera will integrate, during one frame time $t_{c a m}$, this signal :

$$
I_{\text {cam }}=\int_{0}^{t_{c} a m} I_{0} e^{j f_{r n d}(t-z / v)} e^{-j f_{r n d}(t-\tau)} d t
$$

As mentioned $f_{r n d}$ is a pseudo random function, therefore so are $\exp \left(j f_{r n d}(t-z / v)\right)$ and $\exp \left(j f_{r n d}(t-\tau)\right)$. The results of the integration in eq. 2 can be interpreted as the auto correlation function of the pseudo random function $\exp \left(j f_{r n d}(t-z / v)\right)$.

If $\exp \left(j f_{r n d}(t-z / v)\right)$ is considered a purely random function, then this autocorrelation is delta function meaning that the signal recorded by the camera, $I_{\text {cam }}$ is zero except when $\tau=z / v$. Our pseudo random signal 
is generating simply by shifting the phase of the US and the optical reference beam modulations back and forth from 0 to $\pi$. In this case the autocorrelation is not a delta function but still a very narrow one. As seen in figure 3 , even for a duration more than two orders of magnitude greater ( $1 \mathrm{~ms}$ compared to $3 \mu \mathrm{s}$ ), the autocorrelation function is narrower and therefore the spatial resolution is 3 times better with a pseudo random modulation than with a pulsed sine modulation.
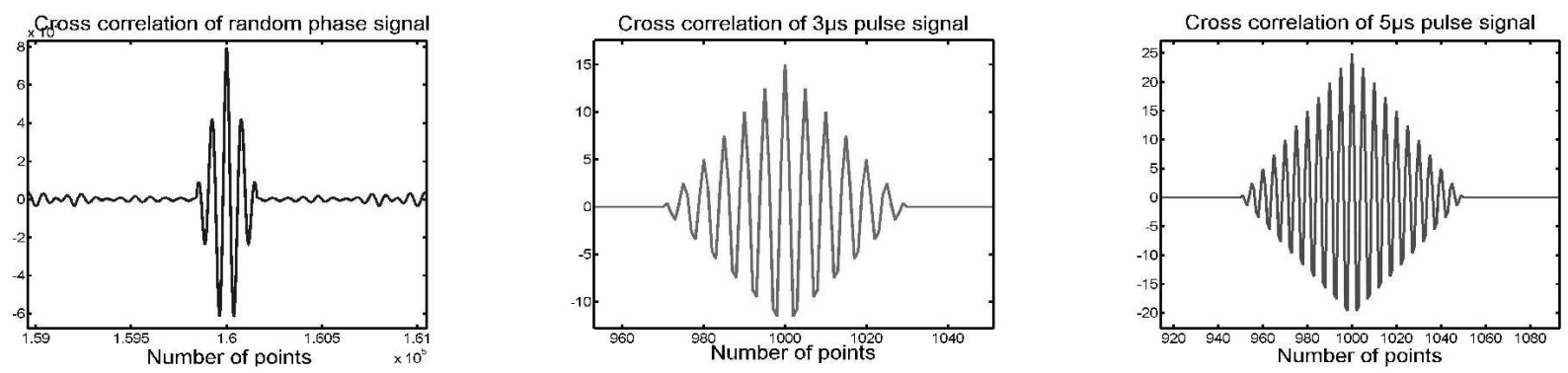

Figure 3. Numerical calculation of the autocorrelation of (left) a $1 \mathrm{~ms}$ pseudo random signal, (center) a $3 \mu$ s sine pulse and (right) a $5 \mu$ s sine pulse

Once again, by adjusting the delay $\tau$ between the acoustic and reference beam signals, we can "select" a specific position along the US propagation axis.

\section{EXPERIMENT}

A $50 \mathrm{~mm}$ thick aquarium is filled with scattering liquid : a diluted solution of intralipid $10 \%$. This solution is then further diluted to vary the optical scttering properties of the liquid. Measurements of the spectral broadening of the diffuse light using the methodology discribed in ref. ${ }^{6}$ as shown are in the range of the $\mathrm{kHz}$, which is comparable to such mesurements made in vivo. ${ }^{6,15}$ The inclusion is a small latex pouch a few milimiters in diameter filled with the same solution dyed with black ink.

Experimental scans along the US propagation axis using the pulsed acoustic and reference beam technique are shown in figures 4 and 5. The signal is plotted in normalized units. As described in ref $^{7}$ our technique allows us th measure the signal and the shot noise simultaneously. We define our normalized signal as (signal shot noise)/shot noise. Figure 4 compares results on two dilutions (3\% and $5 \%$ ) and shows in each casde two scans along the US propagation axis : one over the inclusion (circles) and one away from the inclusion (squares). The experiment is repeated ( 8 times for each scan) to improve the signal to noise ratio. The inclusion is clearly visible even for a small numbe of accumulations. Figure 5 show results on further diluted liquids. As expected the inclusion is more easily seen in the most diluted solution.

Experimental results using the pseudo random modulation technique are shown in figure 6 . The dilution is $5 \%$ and the inclusion is clearly seen in the scan on the left and the $2 \mathrm{D}$ image on the right. The normalized signal level is roughly 5 times higher than the one obtained with the pulse technique, the resolution comparble and the signal to noise ratio is much better, with a single scan.

\section{CONCLUSION}

These results are an important step towards in vivo measurement in which effects of decorrelation of the speckle by movement of, or in, the biological tissues is important. The comparison of the two experimental techniques must be pushed further determine which offers the beast compromise between signal level and resolution while remaining in the safety limits for the amplitude and duration of the US pulses. The setup can and must also be adapted for the concurrent acquisition of the signal at multiple wavelengths, adding spectroscopic information to the images.

\section{ACKNOWLEDGMENTS}

This work has been supported by the Région Ile-de-France, as part of the Cancerôple d'Ile de France. We would also like to acknowledge the support of the Institut National du Cancer. 


\begin{tabular}{ll}
\hline & inclusion \\
$\square$ & no inclusion
\end{tabular}

C $=3 \%, 2$ accumulations

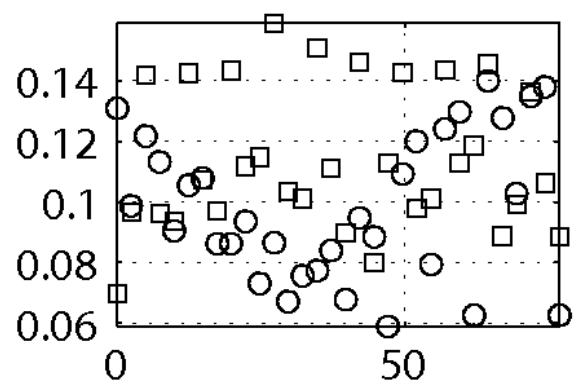

transverse axis ( $\mathrm{mm}$ )

$\mathrm{C}=3 \%, 4$ accumulations

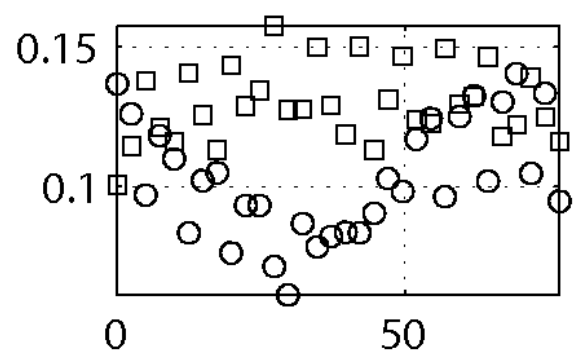

transverse axis ( $\mathrm{mm}$ )

$\mathrm{C}=3 \%, 8$ accumulations

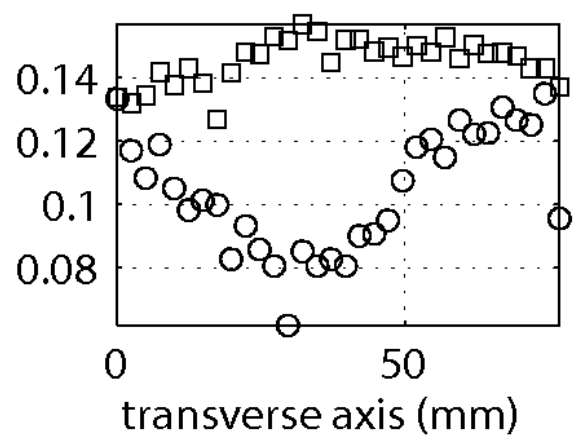

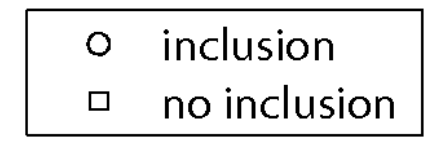

$C=5 \%, 2$ accumulations

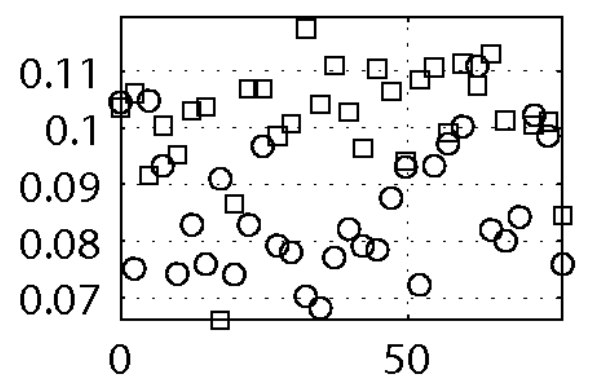

transverse axis ( $\mathrm{mm}$ )

$\mathrm{C}=5 \%, 4$ accumulations

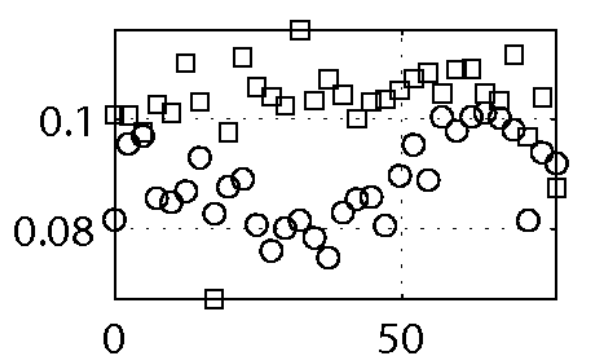

transverse axis (mm)

$\mathrm{C}=5 \%, 8$ accumulations

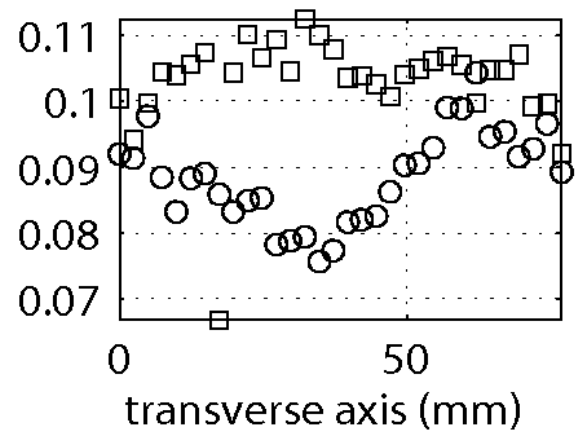

Figure 4. Experimental scans along the US propagation axis using the pulse technique. Results are shown for scan over and away from the inclusion for 2 dilutions of the scattering liquid. Averaging the signal improves the signal to noise ration 


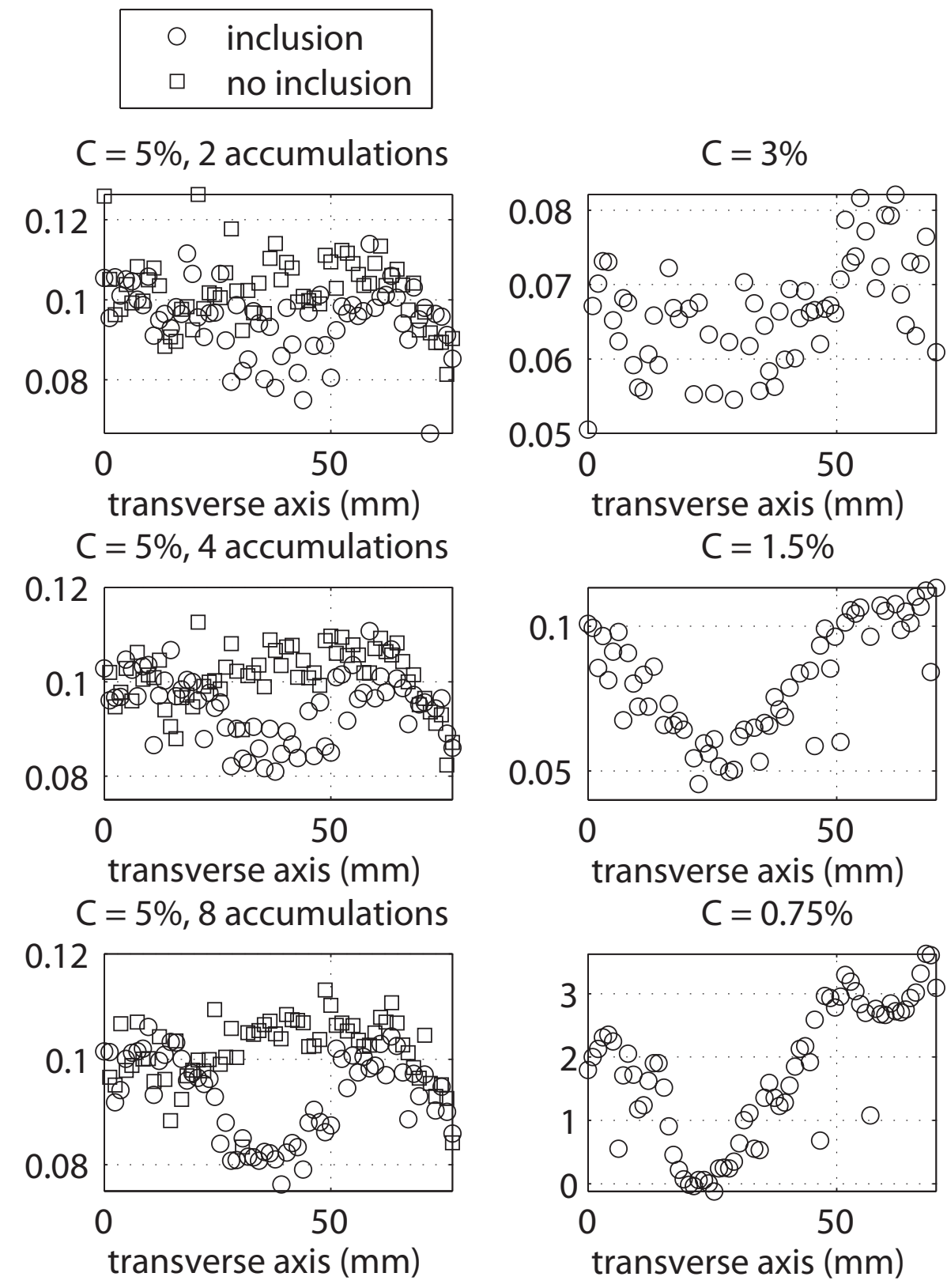

Figure 5. Experimental scans along the US propagation axis using the pulse technique. The inclusion is clearly more easily distinguished as the solution is more diluted, thus less scattering. 

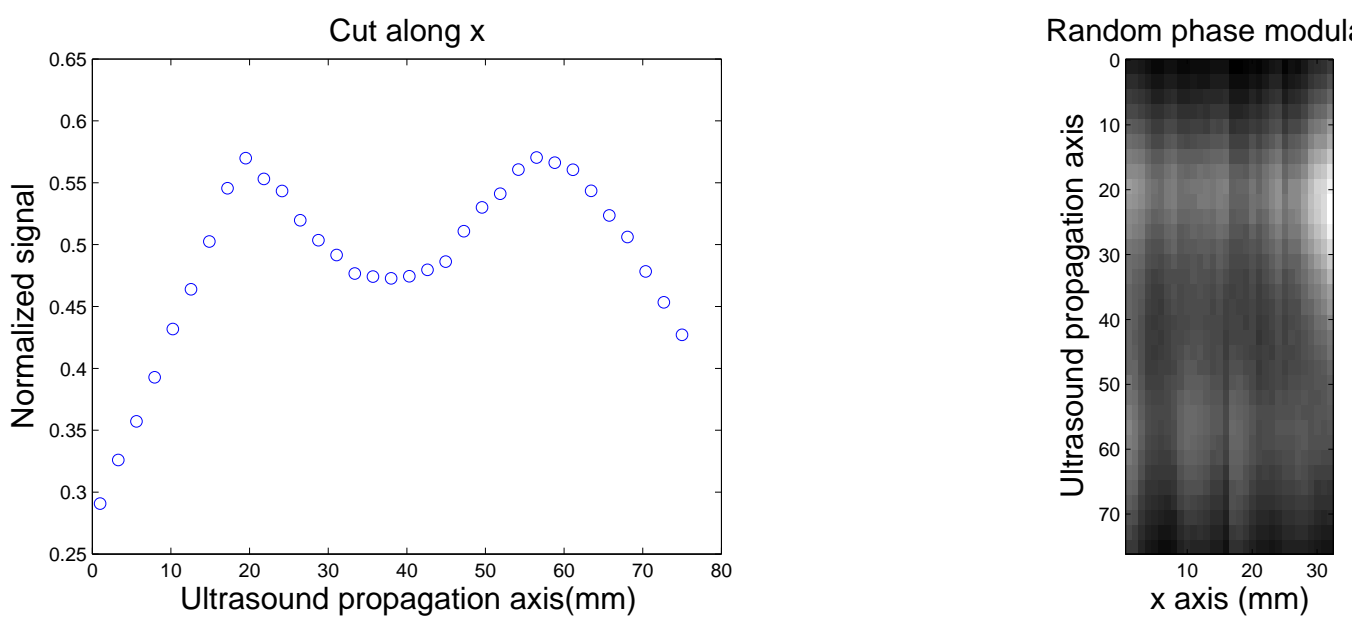

Figure 6. Experimental results using the pseudo random modulation technique.

\section{REFERENCES}

1. L. H. Wang, S. L. Jacques, and X. M. Zhao, "Continuous-wave ultrasonic modulation of scattered laser light to image objects in turbid media," Opt. Lett. 20, p. 629, 1995.

2. W. Leutz and G. Maret, "Ultrasonic modulation of multiply scattered light," Physica B 204(14), 1995.

3. L.-H. Wang, "Mechanisms of ultrasonic modulation of multiply scattered coherent light: an analytic model," Phys. Rev. Lett. 87, pp. 043903-1, 2001.

4. Kempe, M. Larionov, D. Zaslavsky, and A. Z. Genack, "Acousto-optic tomography with multiple scattered light," JOSA 14, pp. 1151-1158, 1997.

5. A. Lev and B. Sfez, "in vivo demonstration of ultrasound-modulated light technique," J. Opt. Soc. Am. A 20, pp. 2347-2354, dec 2003.

6. M. Gross, P. Goy, B. C. Forget, M. Atlan, F. Ramaz, A. C. Boccara, and A. K. Dunn, "Heterodyne detection of multiply scattered monochromatic light with a multipixel detector," Opt. Lett. 30, pp. 13571359 , june 2005.

7. M. Gross, P. Goy, and M. Al-Koussa, "Shot-noise detection of ultrasound-tagged photons in ultrasoundmodulated optical imaging," Opt. Lett. 28, pp. 2482-84, 15 Dec. 2003.

8. M. Atlan, B. C. Forget, F. Ramaz, A. C. Boccara, and M. Gross, "Pulsed acousto-optic imaging with a digital holography scheme," in Photons Plus Ultrasound: Imaging and Sensing 2005, A. A. Oraevsky and L. V. Wang, eds., Poc. SPIE 5697, pp. 209-219, 2005.

9. M. Atlan, B. C. Forget, F. Ramaz, A. C. Boccara, and M. Gross, "Pulsed acousto-optic imaging in dynamic scattering media with heterodyne parallel speckle detection," Opt. Lett. 30, pp. 1360-1362, june 2005.

10. S. Lévêque, A. C. Boccara, M. Lebec, and H. Saint-Jalmes, "Ultrasonic tagging of photon paths in scattering media: parallel speckle modulation processing," Opt. Lett. 24(3), p. 181, 1999.

11. F. Leclerc, L. Collot, and M. Gross, "Numerical heterodyne holography with two-dimensional photodetector arrays," Opt. Lett. 25, p. 716, 2000.

12. L. V. Wang and G. Ku, "Frequency-swept ultrasound-modulated optical tomography of scattering media," Opt. Lett. , 15 June 1998.

13. Gang Yao, Shuliang Jiao, and Lihong V. Wang, "Frequency-swept ultrasound-modulated optical tomography in biological tissue by use of parrallel detection," Opt. Lett. 25, p. 734, 15 May 2000.

14. B. C. Forget, F. Ramaz, M. Atlan, J. Selb, and A. C. Boccara, "High contrast FFT acousto-optical tomography of phantom tissues with a frequency chirp modulation of the ultrasound," Appl. Opt. 42, p. 1379, 1 Mar. 2003.

15. A. Lev and B. Sfez, "Pulsed ultrasound-modulated light tomography," Opt. Lett. 28, pp. 1549-51, 1 Sept. 2003. 
16. P. Santos, M. Atlan, B. C. Forget, F. Ramaz, A. C. Boccara, and M. Gross, "Acousto-optiqc imaging with a digital holography scheme : new scheme to obtain axial resolution," in Novel Optical Instrumentation for Biomedical Application II, C. Depeusinge, ed., Proc. SPIE 5864, 2005. 